УДК 004.652.6

\title{
ОБЛАСТИ ОПРЕДЕЛЕНИЯ РЕЛЯЦИОННЫХ ОПЕРАЦИЙ В БАЗАХ ДАННЫХ ПРИ НАЛИЧИИ НЕОПРЕДЕЛЕННЫХ ЗНАЧЕНИЙ
}

\author{
М. Ю. Выплов
}

\begin{abstract}
Рассматривается структура реляционной базы данных, в которой допускается наличие неопределенных значений, интерпретируемых как "значение не существует". В рамках этой модели изучается формальное построение областей определения для таких объектов, как множество атрибутов, функциональная зависимость, базовые операции реляционной алгебры. Исследуется свойство дистрибутивности областей определений для отдельных комбинаций операций при запросах к БД: показано, что дистрибутивность выполняется для естественного соединения и проекции, но имеет место не во всех случаях для естественного соединения и селекции.
\end{abstract}

Ключевые слова: база данных, реляционная алгебра, область определения, неопределенные значения.

M. Yu. Vyplov. Domains of relational database operations in the presence of uncertain values.

We consider the structure of a relational database in which uncertain values are allowed. These values are interpreted as nonexistent. We study the formal construction of domains for objects such as the set of attributes, functional dependence, and basic operations of relational algebra. The distributive property of the domains is studied for particular combinations of operations for database queries. It is shown that distributivity holds for a natural join and projection but not in all cases for a natural join and selection.

Keywords: database, relational algebra, domain, uncertain values.

MSC: $68 \mathrm{P} 15$

DOI: $10.21538 / 0134-4889-2019-25-1-5-10$

\section{Введение}

Одной из актуальных теоретических проблем реляционной модели данных, до сих пор не имеющей удовлетворительного решения, являются неопределенные значения. Проблема представления неполной информации сказывается на всех видах зависимостей, возникающих при проектировании баз данных (БД). На практике используются специальные маркеры, называемые $N u l l$-значениями (не являющиеся реальными значениями в обычном смысле этого термина). Им соответствуют элементы кортежа, которые представляют неизвестные, неуместные или противоречивые значения. Этот подход неоднократно подвергался критике; известно, что при допущении неопределенных значений возникают трудности как теоретического, так и практического плана.

Среди возможных интерпретаций неопределенных значений можно отметить, например, "отсутствующие", "отсутствующие в силу неприменимости", "неизвестные в настоящее время". Серьезное внимание проблеме неполной информации было уделено в работе [1], где интерпретация неопределенностей сводится к двум видам: 1) значение, возможно, существует, но оно не известно; и 2) значение не существует (ni, "not information"). Для случая $n i$ в [1] предлагается расширение реляционной алгебры, кроме того, делается вывод, что значение $\mathrm{Null}$ не может быть отождествлено с каким-либо другим значением, в том числе само с собой.

Как пример дальнейшего развития исследования неопределенностей и расширенных аксиоматизаций зависимостей можно отметить работы [2;3], в которых, кроме того, приводится и анализируется обширный список трудов по данному направлению.

Естественным требованием к расширениям реляционной модели является обобщение: исключение неопределенных значений должно сводить к классической теории, чему не вполне 
удовлетворяют существующие разработки. В работе С. В. Зыкина "Области определения функциональных зависимостей в базах данных" (Тр. Ин-та математики и механики УрО РАН, 2016, Т. 22, № 3) было предложено оригинальное решение этой проблемы, основанное на областях определения зависимостей (доменах), где nov ("no value") - маркер значений, про которые известно, что они не существуют. Мы также будем придерживаться этой предметной области и обозначений, рассматривая домены различных объектов реляционной модели, и поставим целью анализ свойств операций реляционной алгебры.

Пусть $R\left(A_{1}, A_{2}, \ldots, A_{n}\right)$ - схема отношения реляционной БД, а $X$ и $Y$ - подмножества $\left\{A_{1}, A_{2}, \ldots, A_{n}\right\}$. Говорят, что $X$ функиионально определяет $Y$ или Y функционально завиcum om $X$, и обозначают это как $X \rightarrow Y$, если в любом отношении $r$, являющемся текущим значением $R$, не могут содержаться два кортежа, компоненты которых совпадают по всем атрибутам, принадлежащим множеству $X$, но не совпадают по одному или более атрибутам, принадлежащим множеству $Y$, т. е.

$$
X \rightarrow Y \quad \Leftrightarrow \quad \forall t_{1}, t_{2} \in r \quad t_{1}[X]=t_{2}[X] \Rightarrow t_{1}[Y]=t_{2}[Y] .
$$

Областью определения $\operatorname{dom}(X)$ подмножества атрибутов $X$ в $R$ является множество кортежей $T_{r}(X)=\left\{t_{1}, t_{2}, \ldots, t_{s}\right\} \subseteq r$ текущей реализации $r$, для которых выполнено условие $t[X] \not \supset$ nov.

Областью определения зависимости $\operatorname{dom}(X \rightarrow Y)$ в текущей реализации $r$ является множество кортежей $T_{r}(X \rightarrow Y)=\left\{t_{1}, t_{2}, \ldots, t_{m}\right\} \subseteq r$, для которых выполнены следующие условия:

1) $t[X Y] \not \supset$ nov;

2) для любой пары кортежей $t_{1}, t_{2}$ если $t_{1}[X]=t_{2}[X]$, то $t_{1}[Y]=t_{2}[Y]$.

Null - маркер, присваеваемый любым неопределенным значениям, про которые известно, что эти значения есть, но они пока неизвестны. nov служит заменителем значений, про которых известно, что их нет для какого-либо класса объектов (см. работу С. В.Зыкина). Предполагается, что при правильной декомпозиции отношений этот маркер не появится ни в одном из отношений БД. При плохом проектировании nov может появиться как $\mathrm{Null}$.

П р и м е р 1. БД отдела кадров:

$A_{1}$ - № сотрудника, $A_{2}$ - ФИО сотрудника, $A_{3}$ - должность сотрудника, $A_{4}$ - дата увольнения сотрудника.

Имеем множество функциональных зависимостей $D E P=\left\{A_{1} \rightarrow A_{2}, A_{1} \rightarrow A_{3}, A_{1} \rightarrow A_{4}\right\}$. Тогда $\operatorname{dom}\left(A_{1} \rightarrow A_{4}\right)=\{$ уволенные сотрудники $\}$.

Существует подмножество выражений реляционной алгебры, используемых в языке запросов очень часто и важных для рассмотрения (см. [4, гл. 4]). Этот класс выражений (конструкция "селекция - проекция - соединение") образуется из операторов

○ селекции (выделение описанного объекта);

о естественного соединения (ассоциирование через связи с каким-либо объектом другого типа), ;

○ проекции (определение печатаемых атрибутов последнего объекта).

Напомним, что проекиией $\pi_{X}(R)$ отношения $R$ на подмножество атрибутов $X$ называется множество $\{t[X]: t \in R\}$. Селекиией (выборкой) $\sigma_{L}(R)$ на отношении $R$ с условием $L$ называется множество кортежей $t$, принадлежащих $R$, таких, что при подстановке в формулу $L$ она становится истинной. Eстественным соединением $R_{1} \bowtie R_{2}$ называется соединение по эквивалентности двух отношений $R_{1}$ и $R_{2}$, выполненное по всем общим атрибутам, из результатов которого исключается по одному экземпляру каждого общего атрибута. Формально $R_{1} \bowtie R_{2}=$

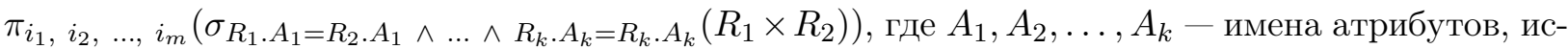
пользуемых и в $R_{1}$, и в $R_{2}, i_{1}, i_{2}, \ldots, i_{m}$ - упорядоченный список всех атрибутов из $R_{1} \times R_{2}$, за исключением $R_{2} \cdot A_{1}, \ldots, R_{2} \cdot A_{k}$.

Построим области определения вышеперечисленных операций при наличии неопределенных значений. Нас будет интересовать, в каких случаях сохраняется дистрибутивность (как 
одно из важнейших свойств для процесса последовательного применения операций различного типа к набору данных).

\section{1. Области определений естественного соединения и проекции}

Будем рассматривать схемы отношений $R_{1}, R_{2}$ на множествах атрибутов $U_{1}, U_{2}$.

О п р е д е л е н и е 1 . Областью определения операции естественного соединения отношений (произвольных реализаций) $R_{1}, R_{2}$ будем называть следующий набор пар кортежей:

$D_{\bowtie}=\operatorname{dom}\left(R_{1} \bowtie R_{2}\right)=\left\{\left(t_{1}, t_{2}\right): t_{1} \in R_{1}, t_{2} \in R_{2}, t_{1}, t_{2} \in \operatorname{dom}\left(U_{1} \cap U_{2}\right), t_{1}\left[U_{1} \cap U_{2}\right] \not \supset\right.$ Null, $\left.t_{2}\left[U_{1} \cap U_{2}\right] \not \supset N u l l\right\}$.

О п р е д е л е н и е 2 . Областью определения операции проекции $\pi_{X}(R)$ отношения $R$ будем называть область определения множества атрибутов $X$, т. е. $D_{\pi}=\operatorname{dom}\left(\pi_{X}(R)\right)=$ $\operatorname{dom}(X)=\{t \in R: t[X] \not \supset n o v\}$.

Утверждение 1. Выполнено свойство дистрибутивности для областей определений естественного соединения и проекиии по множеству $X$ :

$$
\pi_{X}\left(R_{1} \bowtie R_{2}\right)=\pi_{X}\left(\pi_{X_{1}}\left(R_{1}\right) \bowtie \pi_{X_{2}}\left(R_{2}\right)\right),
$$

где $X_{1}=\left(X \cap U_{1}\right) \cup\left(U_{1} \cap U_{2}\right)=U_{1} \cap\left(X \cup U_{2}\right), X_{2}=\left(X \cap U_{2}\right) \cup\left(U_{1} \cap U_{2}\right)=U_{2} \cap\left(X \cup U_{1}\right)$.

Д о к а з а т е л ь с т в о. Действительно, можно проверить, раскрывая скобки, что обе части (1.1) равны $\left\{\left(t_{1}|| t_{2}\right)[X]: t_{1} \in R_{1}, t_{2} \in R_{2}, t_{1}\left[X_{1}\right] \not \supset\right.$ nov, $t_{2}\left[X_{2}\right] \not \supset$ nov, $t_{1}\left[U_{1} \cap U_{2}\right]=$ $\left.t_{2}\left[U_{1} \cap U_{2}\right] \not \supset N u l l\right\}$, где $t_{1} \| t_{2}-$ склейка кортежей $t_{1}$ и $t_{2}$ (кортеж, составленный из всех элементов $t_{1}$ и $t_{2}$, относящихся к различным атрибутам).

Проверим также, что левая и правая части (1.1) имеют общую область определения.

Найдем область определения для выражения из левой части (1.1), последовательно рассматривая операции проекции и естественного соединения:

$\operatorname{dom}\left(\pi_{X}\left(R_{1} \bowtie R_{2}\right)\right)=D_{\bowtie}\left(\left\{t \in R_{1} \bowtie R_{2}: t[X] \not \supset n o v\right\}\right)=\left\{\left(t_{1}, t_{2}\right): t_{1} \in R_{1}, t_{2} \in R_{2}\right.$, $\left(t_{1}|| t_{2}\right)[X] \not \ngtr$ nov, $\left.t_{1}, t_{2} \in \operatorname{dom}\left(U_{1} \cap U_{2}\right), t_{1}\left[U_{1} \cap U_{2}\right] \not \supset N u l l, t_{2}\left[U_{1} \cap U_{2}\right] \not \supset N u l l\right\}=\left\{\left(t_{1}, t_{2}\right):\right.$ $t_{1} \in R_{1}, t_{2} \in R_{2}, t_{1}, t_{2} \in \operatorname{dom}\left(X \cup\left(U_{1} \cap U_{2}\right)\right), t_{1}\left[U_{1} \cap U_{2}\right] \not \supset$ Null, $\left.t_{2}\left[U_{1} \cap U_{2}\right] \not \supset N u l l\right\}=$ $\left\{\left(t_{1}, t_{2}\right): t_{1} \in R_{1} \cap \operatorname{dom}\left(X_{1}\right), t_{2} \in R_{2} \cap \operatorname{dom}\left(X_{2}\right), t_{1}\left[U_{1} \cap U_{2}\right] \not \supset N u l l, t_{2}\left[U_{1} \cap U_{2}\right] \not \supset N u l l\right\}$.

Аналогично найдем область определения для правой части (1.1):

$\operatorname{dom}\left(\pi_{X}\left(\pi_{X_{1}}\left(R_{1}\right) \bowtie \pi_{X_{2}}\left(R_{2}\right)\right)=D_{\bowtie}\left(t \in \pi_{X_{1}}\left(R_{1}\right) \bowtie \pi_{X_{2}}\left(R_{2}\right): t[X] \not \supset n o v\right\}\right)=D_{\pi}\left(\left\{\left(t_{1}, t_{2}\right):\right.\right.$ $\left.\left.t_{1} \in \pi_{X_{1}}\left(R_{1}\right), t_{2} \in \pi_{X_{2}}\left(R_{2}\right), t_{1}, t_{2} \in \operatorname{dom}\left(X \cup\left(U_{1} \cap U_{2}\right)\right), t_{1}\left[U_{1} \cap U_{2}\right] \not \supset N u l l, t_{2}\left[U_{1} \cap U_{2}\right] \not \supset N u l l\right\}\right)=$ $\left\{\left(t_{1}, t_{2}\right): t_{1} \in R_{1} \cap \operatorname{dom}\left(X_{1}\right), t_{2} \in R_{2} \cap \operatorname{dom}\left(X_{2}\right), t_{1}\left[U_{1} \cap U_{2}\right] \not \supset N u l l, t_{2}\left[U_{1} \cap U_{2}\right] \not \supset N u l l\right\}$.

Как можно убедиться, полученные выражения областей определения совпадают.

3 а м е ч а н и е. Представленные формулировки также верны для операции декартова произведения $R_{1} \times R_{2}$, если ее рассматривать как частный случай естественного соединения по пустому множеству атрибутов.

\section{2. Область определения селекции}

Будем рассматривать реляционную базу данных со схемой $R=\left\{R_{1}, R_{2}, \ldots, R_{N}\right\}$ и логическую формулу (предикат) $L$ над $R$, выполнение которого зависит от значений множества атрибутов $X \subseteq U$. Для этого множества введем обозначение $[L]$.

Выделим элементарные выражения, над которыми будут осуществляться логические операции $\vee, \&, \neg$.

Логические формулы, по которым проводится селекция, будем рассматривать в дизъюнктивной нормальной форме. В общем случае формула $L$ имеет вид

$$
L=K_{1} \vee K_{2} \vee \ldots \vee K_{l}, \quad K_{i}=E_{1} \& E_{2} \ldots \& E_{s}, \quad i=1, \ldots, l,
$$


где $E_{j}, j=1, \ldots, s$ - элементарные выражения, в которых явным образом специфицированы расширенные имена атрибутов $R_{i} \cdot A_{j}$ (атрибут $A_{j}$ в отношении $R_{i}$ ).

Возможные значения $E_{j}$ :

○ операция сравнения $\operatorname{Expr}_{1} \theta \operatorname{Expr}_{2}, \theta$ - оператор сравнения $(\theta \in\{=, \neq,>,<, \leq, \geq\})$;

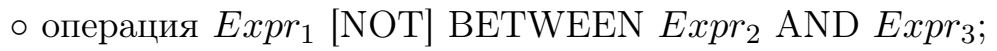

о операция Expr [NOT] IN $S$, где $S$ - список значений либо подзапрос, результатом которого является столбец атрибута $A_{j}$ в отношении $R_{i}$;

○ операция $S t r_{1}$ [NOT] LIKE $S t r_{2}$, где $S t r_{i}$ - строки;

○ операция Expr $\theta$ ALL / ANY $S$.

Здесь $\operatorname{Expr}_{i}$ - согласованные по типам допустимые выражения, определенные на множестве расширенных имен атрибутов и констант.

Предложим подход к формализации области определения селекции, учитывающий область истинности $M(L)$ логической формулы $L$, - область, где $L$ определена и истинна. Следует отметить, что оперирование областями истинности, в частности, играет важную роль при аналитическом сравнении пользовательских запросов, актуальном для оптимизации использовании кэша данных [5].

Рассмотрим множество кортежей, на котором определена операция селекции. Если просто принять в качестве $\operatorname{dom}(L)$ множество $\{t \in \operatorname{dom}([L]): t \not \supset N u l l\}$, то, в зависимости от вида $L$, могут заведомо отбрасываться некоторые кортежи, к которым селекция применима и возвращает однозначный результат.

П р и м е р 2. БД склада магазина:

$A_{1}$ - № товара, $A_{2}$ - наименование товара, $A_{3}$ - количество экземпляров товара в наличии, $A_{4}$ - цена товара. $D E P=\left\{A_{1} \rightarrow A_{2}, A_{1} \rightarrow A_{3}, A_{1} \rightarrow A_{4}\right\}$.

$\operatorname{dom}\left(A_{1} \rightarrow A_{4}\right)=\{$ товары, выставленные в продажу $\}$.

Рассмотрим запрос "Получить перечень названий товаров, имеющихся в количестве менее 10 штук либо стоимостью более 10 тыс. руб." Это проекция на подмножество $X=\left\{A_{2}\right\}$ результата операции селекции с формулой $L=\left(A_{3}<10\right) \vee\left(A_{4}>10000\right)$. Кортежи, для которых первая часть формулы истинна, удовлетворяют запросу независимо от значения атрибута $A_{4}$. Поэтому, чтобы не потерять их (в случае, когда стоимость не определена), придется принимать во внимание области истинности.

О п р е д е л е н и е 3 . Областью определения операции селекции $\sigma_{L}(R)$ будем называть

$$
\operatorname{dom}(L)= \begin{cases}t \in \operatorname{dom}\left(\left[E_{j}\right]\right): t\left[E_{j}\right] \not \supset N u l l, & L \text { содержит единственное } \\ & \text { элементарное выражение } E, \\ \bigcap_{j} \operatorname{dom}\left(E_{j}\right), & L \text { содержит единственный } \\ \bigcup_{i} M\left(K_{i}\right) & \text { конъюнкт } K=E_{1} \& E_{2} \& \ldots \& E_{s}, \\ \text { иначе. }\end{cases}
$$

Проверим, выполняется ли свойство дистрибутивности для областей определений естественного соединения и селекции:

$$
\sigma_{L}\left(R_{1} \bowtie R_{2}\right)=\sigma_{L_{1}}\left(R_{1}\right) \bowtie \sigma_{L_{2}}\left(R_{2}\right),
$$

где $L_{1}$ и $L_{2}-$ составные части логической формулы $L$, такие что $\left[L_{1}\right] \subseteq U_{1},\left[L_{2}\right] \subseteq U_{2}$. Пусть $L_{1}=K_{11} \vee K_{12} \vee \ldots \vee K_{1 l_{1}}, L_{2}=K_{21} \vee K_{22} \vee \ldots \vee K_{2 l_{2}}$. Рассмотрим варианты, типичные для запросов к БД: будем считать, что $L$ может представлять собой либо дизъюнкцию, либо конъюнкцию $L_{1}$ и $L_{2}$ (допустимо также отрицание, но будем считать, что оно содержится внутри конъюнктов).

Утверждение 2. Свойство дистрибутивности (2.1)

1) отсутствует в случае $L=L_{1} \vee L_{2}$; 
2) имеет место (относительно результата операций) в случае $L=L_{1} \& L_{2}$, при этом относительно области определения выполняется, только если формулы $L_{1}$ и $L_{2}$ либо обе состоят из единственного конгюнкта, либо обе не состоят.

Д о к а з а т е л ь с т в о. 1) Пусть $L=L_{1} \vee L_{2}$. Считаем, что $L_{1}$ и $L_{2}$ не являются тождественно равными TRUE или FALSE (т. е. в формулах отсутствуют тривиальные условия на атрибуты, например, условия вида $E x p r_{1}=\operatorname{Expr}_{1}$ и сводящиеся к ним). Таким образом, множества $M\left(L_{1}\right), \operatorname{dom}\left(L_{1}\right) \backslash M\left(L_{1}\right), M\left(L_{2}\right), \operatorname{dom}\left(L_{2}\right) \backslash M\left(L_{2}\right)$ непусты. Рассмотрим кортежи $t_{11} \in R_{1} \cap M\left(L_{1}\right), t_{12} \in R_{1} \cap \operatorname{dom}\left(L_{1}\right) \backslash M\left(L_{1}\right), t_{21} \in R_{2} \cap M\left(L_{2}\right), t_{22} \in R_{2} \cap \operatorname{dom}\left(L_{2}\right) \backslash M\left(L_{2}\right)$. В то время, как $\sigma_{L}\left(R_{1} \bowtie R_{2}\right)$ включает кортежи $t_{11}\left\|t_{21}, t_{12}\right\| t_{21}, t_{11} \| t_{22}$, соединение $\sigma_{L_{1}}\left(R_{1}\right) \bowtie$ $\sigma_{L_{2}}\left(R_{2}\right)$ содержит только кортеж $t_{11} \| t_{21}$. Очевидно, дистрибутивность отсутствует.

2) Пусть $L=L_{1} \& L_{2}$. Согласно определению имеем

$$
\begin{aligned}
& \operatorname{dom}\left(L_{1}\right)= \begin{cases}t \in \operatorname{dom}\left(\left[K_{11}\right]\right), & l_{1}=1, \\
\bigcup_{i} M\left(K_{1 i}\right) & \text { иначе, }\end{cases} \\
& \operatorname{dom}\left(L_{2}\right)= \begin{cases}t \in \operatorname{dom}\left(\left[K_{21}\right]\right), & l_{2}=1, \\
\bigcup_{j} M\left(K_{2 j}\right) & \text { иначе, }\end{cases} \\
& \operatorname{dom}(L)= \begin{cases}t \in \operatorname{dom}([K]), \text { где } K=K_{11} \& K_{21}, & l_{1}=l_{2}=1, \\
\bigcup_{i, j} M\left(K_{1 i} \& K_{2 j}\right) & \text { иначе. }\end{cases}
\end{aligned}
$$

Дистрибутивность (относительно результата операций) имеет место. Действительно, можно проверить, что обе части (2.1) равны

$$
\begin{gathered}
\left\{\left(t_{1}, t_{2}\right): t_{1} \in R_{1}, t_{2} \in R_{2}, t_{1}\left[U_{1} \cap U_{2}\right]=t_{2}\left[U_{1} \cap U_{2}\right] \not \supset N u l l,\left(t_{1}|| t_{2}\right) \in M(L)=\bigcup_{i, j} M\left(K_{1 i} \& K_{2 j}\right)\right\} \\
=\left\{\left(t_{1}, t_{2}\right): t_{1} \in R_{1}, t_{2} \in R_{2}, t_{1}\left[U_{1} \cap U_{2}\right]=t_{2}\left[U_{1} \cap U_{2}\right] \not \supset N u l l, t_{1} \in M\left(L_{1}\right), t_{2} \in M\left(L_{2}\right)\right\} .
\end{gathered}
$$

Можно видеть, что при этом относительно области определения дистрибутивность наличествует, если формулы $L_{1}$ и $L_{2}$ либо обе состоят из единственного конъюнкта, либо обе не состоят. Покажем, что в ином случае области не совпадают. Пусть без ограничения общности $l_{1}>1, l_{2}=1$. Тогда область определения левой части равенства (2.1) принимает вид $M\left(K_{21}\right) \cap \bigcup_{i} M\left(K_{1 i}\right)$, а правой части $-\operatorname{dom}\left(K_{21}\right) \cap \bigcup_{i} M\left(K_{1 i}\right)$.

П р и м е р 3. Рассмотрим запрос к БД склада из примера 2 , в котором одна часть состоит из единственного конъюнкта, а другая - из нескольких. Пусть $\left[R_{1}\right] \supseteq A_{2} \cup A_{3},\left[R_{2}\right] \supseteq A_{4}$, и мы запрашиваем номера товаров, удовлетворяющих следующему условию $L: L=L_{1} \& L_{2}$, $L_{1}=\left(\left(A_{2}=\right.\right.$ "Принтер") $\left.\vee\left(A_{3}<10\right)\right), L_{2}=\left(A_{4}>10000\right)$. Тогда область определения правой части (2.1) включает кортежи со значениями атрибута $A_{4}$, меньшими или равными 10000 , а область определения левой части - не включает. Это обосновывается удачным разложением формулы: рассматривая ее по частям, можем выполнять меньше действий для нахождения области определения (в отличие от области истинности).

\section{Заключение}

Классическая теория БД подразумевает, что области определения операций совпадают со всей прикладной областью. В данной статье показано, что это не так, и у операций также имеются свои области определения, зависящие от содержания данных и условий, накладываемых на них.

При предлагаемом подходе выдаются данные только из области определения запроса, в котором нет неопределенных значений для запрошенных атрибутов. Стандартный SQL-запрос может быть преобразован к необходимому виду по правилам, предложенным в статье [6]. 


\section{СПИСОК ЛИТЕРАТУРЫ}

1. Zaniolo C. Database relations with null values // J. Comput. System Sci. 1984. no. 28. P. $142-166$. doi: 10.1016/0022-0000(84)90080-1.

2. Hartmann S., Link S. The implication problem of data dependencies over SQL table definitions: axiomatic, algorithmic and logical characterizations // ACM Transactions on Database Systems. 2012. Vol. 37, no. 2. P. 1-40. doi: 10.1145/2188349.2188355.

3. Kohler H., Link S. Inclusion dependencies and their interaction with functional dependencies in SQL // J. Comput. Syst. Sci. 2017. Vol. 85. P. 104-131. doi: 10.1016/j.jcss.2016.11.004 .

4. Ульман Дж. Основы систем баз данных. М.: Финансы и статистика, 1983. 334 с.

5. Мосин С.В., Зыкин С.В. Кэширование запросов к реляционной базе данных с использованием областей истинности // Моделирование и анализ информ. систем. 2015. Т. 22, № 2. С. 248-258. doi: 10.18255/1818-1015-2015-2-248-258 .

6. Зыкин С.В., Полуянов А.Н. Формирование многомерных данных с использованием промежуточных представлений // Проблемы управления. 2013. № 5. С. 54-59.

Поступила 30.03.2018

После доработки 24.09.2018

Выплов Михаил Юрьевич Принята к публикации 14.10.2018

канд. физ.-мат. наук, науч. сотрудник

Институт математики им. С.Л.Соболева СО РАН,

г. Новосибирск

e-mail: vyplov@ofim.oscsbras.ru

\section{REFERENCES}

1. Zaniolo C. Database relations with null values. J. Comput. System Sci., 1984, no. 28, pp. 142-166. doi: 10.1016/0022-0000(84)90080-1.

2. Hartmann S., Link S. The implication problem of data dependencies over SQL table definitions: axiomatic, algorithmic and logical characterizations. ACM Transactions on Database Systems, 2012, vol. 37 , no. 2 , pp. $1-40$. doi: $10.1145 / 2188349.2188355$.

3. Kohler H., Link S. Inclusion dependencies and their interaction with functional dependencies in SQL. J. Comput. Syst. Sci. 2017, vol. 85, pp. 104-131. doi: 10.1016/j.jcss.2016.11.004 .

4. Ullman J. Principles of Database Systems. Potomac, Maryland: Computer Science Press, 1980, 379 p. ISBN: 0-914894-13-7. Translated to Russian under the title Osnovy sistem baz dannykh. Moscow, Finansy i statistika Publ., 1983, 334 p.

5. Mosin S.V., Zykin S.V. Truth space method for caching database queries. Model. Anal. Inform. Sist., 2015, vol. 22, no. 2, pp. 248-258 (in Russian). doi: 10.18255/1818-1015-2015-2-248-258 .

6. Zykin S.V., Poluyanov A. N. Formation of multidimensional data using intermediate representations. Probl. Upr., 2013, no. 5, pp. 54-59 (in Russian).

Received March 30, 2018

Revised September 24, 2018

Accepted October 14, 2018

Mikhail Yur'evich Vyplov, Cand. Sci. (Phys.-Math.), Sobolev Institute of Mathematics of the Siberian Branch of the Russian Academy of Sciences, Novosibirsk, 630090 Russia, e-mail: vyplov@ofim.oscsbras.ru . 